\title{
Dimensões de burnout como preditoras da tensão emocional e depressão em profissionais de enfermagem em um contexto hospitalar
}

\author{
Dimensions of burnout as predictors of emotional stress and \\ depression of nursing professionals in a hospital context
}

\author{
Danielle Figueiredo Patrício' (D), Silvânia da Cruz Barbosa², Renata Pimentel da Silva, \\ Rafaela Ferreira da Silva ${ }^{4}$ \\ 'Programa de Pós-graduação em Psicologia da Saúde, Universidade Estadual da Paraíba (UEPB) - Campina Grande (PB), Brasil. \\ 2Programa de Pós-graduação em Psicologia da Saúde, Departamento de Psicologia, Universidade Estadual da Paraíba (UEPB) - \\ Campina Grande (PB), Brasil. \\ ${ }_{3}^{3}$ Programa de Pós-graduação em Psicologia Social, Universidade Federal da Paraíba (UFPB) - João Pessoa (PB), Brasil. \\ ${ }^{4}$ Programa de Pós-graduação em Psicologia da Saúde, Universidade Estadual da Paraíba (UEPB) - Campina Grande (PB), Brasil.
}

Como citar: Patrício DF, Barbosa SC, Silva RP, Silva RF. Dimensões de burnout como preditoras da tensão emocional e depressão em profissionais de enfermagem em um contexto hospitalar. Cad Saúde Colet, 2021;29(4):575-584. https://doi. org/10.1590/1414-462X202129040441

\begin{abstract}
Resumo
Introdução: Síndrome de burnout (SB) é um distúrbio emocional caracterizado por extremo esgotamento físico e mental decorrente de situações laborais estressantes. Já depressão é uma alteração afetiva decorrente de experiências pessoais negativas, sem referência a contextos específicos. Objetivo: Analisar possível associação entre burnout e tensão emocional e depressão em profissionais de enfermagem de um hospital em Campina Grande, Paraíba. Método: O delineamento foi quantitativo-correlacional, sendo "tensão emocional e depressão" a variável dependente e os fatores de burnout ("exaustão emocional" - EE; "cinismo" - Cl; "ineficácia no trabalho" - IT) variáveis independentes, não pressupondo relações de causalidade. Participaram 220 sujeitos. Foram aplicados os instrumentos MBI (HSS), QSG-12 e Ficha Sociodemográfica. Foram efetuadas análises descritivas, de correlação (Spearman) e de regressão. Resultados: Da amostra, 15\% apresentaram elevada EE, 8,6\%, moderada tensão, e 3,2\%, depressão. Foi observada predição compartilhada entre os três fatores da SB com a "tensão emocional e depressão", sendo EE o mais forte preditor, explicando $17 \%$ da variância. Conclusão: Os dados oferecem forte sugestão de que elevados níveis de EE influenciam a depressão, sendo imprescindível enfrentá-los e oferecer suporte psicológico, educativo e material para a recomposição da energia física e mental sugada pela sobrecarga laboral.
\end{abstract}

Palavras-chave: burnout; tensão emocional; depressão; enfermagem.

\begin{abstract}
Background: Burnout Syndrome (BS) is an emotional disorder characterized by extreme physical and mental exhaustion resulting from stressful work situations; depression is an affective alteration resulting from negative personal experiences, without reference to specific contexts. Objective: to analyze a possible association between burnout and emotional tension and depression in nursing professionals at a hospital in Campina Grande-PB. Method: The design was quantitative-correlational, with Emotional Tension and Depression as the dependent variable and the burnout factors (Emotional
\end{abstract}

Este é um artigo publicado em acesso aberto (Open Access) sob a licença Creative Commons Attribution, que permite uso, distribuição e reprodução em qualquer meio, sem restrições desde que o trabalho original seja corretamente citado.

\footnotetext{
Trabalho realizado em instituição hospitalar privada no município de Campina Grande (PB), Brasil.

Correspondência: Danielle Figueiredo Patrício. E-mail: dani.enfermagem8@gmail.com

Fonte de financiamento: nenhuma.

Conflito de interesses: nada a declarar.

Recebido em: Set. 03, 2018. Aprovado em: Set. 06, 2020
} 
Exhaustion - EE, Cynicism - Cl, Ineffectiveness at Work - IT) independent variables, not assuming causal relationships. The participants were 220 individuals The instruments MBI (HSS), QSG-12, and Sociodemographic Record were applied. Descriptive, correlation (Spearman), and regression analyzes were performed. Results: $15 \%$ of the sample shows high EE, $8.6 \%$ moderate tension, and $3.2 \%$ depression. There was a shared prediction between the three factors of BS with Emotional Tension and Depression, with EE being the strongest predictor, explaining $17 \%$ of the variance. Conclusion: The data offer a strong suggestion that high levels of EE influence depression, and it is essential to face them by offering psychological, educational, and material support to restore the physical and mental energy caused by work overload.

Keywords: burnout; emotional tension; depression; nursing.

\section{INTRODUÇÃO}

Trabalhar em condições deletérias pode provocar agravos na saúde física e psíquica. Embora a conexão entre saúde e condições de trabalho não seja um tema recente, continua atualizado, relevante e bastante discutido nas pesquisas ${ }^{2}$.

Nos estudos que relacionam trabalho e saúde, são recorrentes aqueles que focalizam o profissional da enfermagem ${ }^{3-5}$. O trabalho de enfermagem no ambiente hospitalar possui características bastante peculiares, tais como: estabelecer contato íntimo com pacientes e familiares em situações-limite de dor, sofrimento e/ou morte, atuar em equipe multidisciplinar, suportar jornadas extensas de trabalho, horários rodiziados e plantão, lidar com superlotação hospitalar, com insuficiência de recursos materiais e humanos, com baixos salários e com baixa valorização social da profissão. Tais características, associadas a um ambiente hospitalar enquanto lugar insalubre e propício ao adoecimento, podem resultar no desgaste físico e emocional do enfermeiro ${ }^{6,7}$.

Por causa dos baixos salários da categoria, geralmente os profissionais de enfermagem têm mais de um vínculo empregatício, podendo implicar a diminuição de horários de alimentação, lazer, repouso, sono, convívio social e familiar e, consequentemente, sobrecarga física e mental. Em conjunto, esses aspectos tornam-se fatores de desgaste e sofrimento, podendo conduzir ao aparecimento de distúrbios psíquicos menores (não psicóticos), entre os quais burnout e depressão6,8.

Burnout é uma síndrome que surge como uma resposta do indivíduo à tensão emocional crônica no trabalho, sendo formada por três dimensões interdependentes: "exaustão emocional" (EE), que se expressa por esgotamento físico e mental decorrente de sobrecarga no trabalho; "cinismo" $(\mathrm{Cl})$, que se expressa pelo endurecimento afetivo nas relações interpessoais no trabalho; e "ineficácia no trabalho" (IT), que se expressa por sentimento de frustração e descontentamento com o desempenho no trabalho ${ }^{9-11}$. Acomete, geralmente, os indivíduos mais motivados no trabalho, sem psicopatologias prévias e com uma história de desempenho satisfatório $^{12,13}$.

O desenvolvimento de burnout ocorre por um processo lento, gradativo e, muitas vezes, imperceptível pelo indivíduo acometido ${ }^{14}$, podendo, inclusive, levar meses ou anos para ser corretamente diagnosticado por profissionais da saúde, visto que seus diferentes sintomas (físicos, cognitivos, comportamentais e emocionais) podem ser confundidos com outros distúrbios psíquicos, entre os quais a depressão.

A depressão é um transtorno afetivo, geralmente caracterizado por um estado de humor triste, apatia, redução do interesse, capacidade e/ou prazer em realizar as atividades cotidianas, ansiedade, desesperança, alterações de sono (insônia ou hipersonia) e de apetite (perda ou ganho de peso $)^{14-17}$. Os fatores desencadeantes variam de pessoa para pessoa e são relativos a fatores intrínsecos (sexo, idade, vulnerabilidade genética e aspectos neuroendócrinos) e extrínsecos (suporte e renda familiar, fatores ambientais e interações sociais). Atualmente, é considerado um dos distúrbios mais incapacitantes para os trabalhadores, ocasionando altos índices de absenteísmo e afastamentos prolongados (licenças médicas), gerando custos às organizações e aos cofres públicos ${ }^{18}$.

De modo geral, a pessoa deprimida apresenta desânimo e descontentamento com a vida, dificuldade de concentração, ideias suicidas, enquanto o indivíduo com burnout 
apresenta sintomas mais ou menos semelhantes, porém relativos às situações de trabalho, ou seja, o indivíduo se sente sugado pelo trabalho e tende a fazer uma avaliação negativa das suas competências profissionais, e não de si mesmo ${ }^{16,19-21}$. Portanto, burnout é um constructo social decorrente das dificuldades nas relações interpessoais e organizacionais, ao passo que a depressão decorre de um conjunto de emoções e cognições que têm consequências sobre as relações interpessoais e organizacionais ${ }^{16,19}$.

Diferentes estudos indicam que burnout e depressão são constructos relacionados ${ }^{22-26}, \mathrm{e}$, embora não haja consenso na literatura sobre qual predispõe ao outro ${ }^{16,27}$, eles parecem estar intrinsecamente ligados em relação à gênese do problema, pois ambos surgem de fatores etiológicos comuns, por exemplo, o estresse crônico. Contudo, são patologias diferentes que apresentam comorbidades, ou seja, podem surgir simultaneamente em um mesmo paciente ${ }^{28}$.

Tomando-os como constructos relacionados, a presente pesquisa analisou a possível associação entre burnout, tensão emocional e depressão em profissionais de enfermagem de um hospital em Campina Grande, Paraíba.

\section{MÉTODO}

A pesquisa se caracteriza como descritivo-exploratória e transversal, com abordagem quantitativa, estabelecendo relações entre variáveis por meio de um recorte único no tempo, porém sem manipulá-las. A instituição hospitalar pesquisada possui 263 profissionais de enfermagem (técnicos e enfermeiros), com vínculo empregatício efetivo.

A amostra foi composta a partir de uma estratégia acidental não probabilística, por conveniência ${ }^{29}$, incluindo o maior número possível de sujeitos ativos no serviço, com no mínimo seis meses de tempo de trabalho no hospital e que concordaram livremente em colaborar com a pesquisa. Foram excluídos os profissionais terceirizados, os que se recusaram a responder aos questionários e os que estavam afastados do trabalho (férias, licença médica, treinamento) ou ausentes durante a coleta dos dados. Com base nesses critérios, foi obtida a participação de 220 sujeitos, sendo 178 técnicos e 42 enfermeiros, equivalente a uma amostra de $83,66 \%$ da população.

Foram utilizados três instrumentos. Um deles foi o Maslach Burnout Inventory - Service Human Survey (MBI-HSS), de autoria de Maslach e Jackson ${ }^{30}$, que corresponde à versão específica para profissionais de saúde. Esse instrumento possui 22 itens distribuídos em uma escala Likert de 7 pontos, variando de 0 (nunca) a 6 (todo dia). Foi traduzido e adaptado para a língua protuguesa ${ }^{31}$ e validado ${ }^{32}$ em uma amostra multifuncional que incluiu profissionais de enfermagem. Em tal validação, foi usado o sistema de pontuação de 1 a 5, que havia sido recomendado por Tamayo ${ }^{33}$ após ter identificado dificuldades dos sujeitos em responder aos itens usando as categorias de frequência de 7 pontos. Contudo, foi feita a opção por seguir as especificidades do modelo original americano, com escala Likert de 7 pontos, que permite aplicar os pontos de corte recomendados por Shirom ${ }^{34}$ nas três subescalas do MBI: EE, Cl e IT, com índices de consistência interna (alfa de Cronbach) de 0,85, 0,65 e 0,94, respectivamente ${ }^{32}$.

O segundo instrumento foi o Questionário de Saúde Geral (QSG-12), elaborado por Goldberg ${ }^{35}$ para medir distúrbios psíquicos menores (de caráter não psicótico). O instrumento conta com versões de 60, 30, 20 e 12 itens distribuídos em escala Likert de 4 pontos, que variam de 0 a 3. Foi feita a opção por aplicar a versão reduzida, de 12 itens, cuja evidência de validade na pesquisa de Borges e Argolo $^{36}$ aponta adequação para mensurar dois fatores: 1) redução da autoeficácia e 2) tensão emocional e depressão, cujos índices de consistência interna (alfa de Cronbach) são de 0,86 e de 0,83, respectivamente. Apenas este último fator foi utilizado na pesquisa para avaliar o grau de esgotamento psíquico e depressão para enfrentar problemas e dificuldades em situações de trabalho.

O terceiro instrumento foi a Ficha Sociodemográfica, para recolher informações relativas ao perfil biográfico (idade, sexo, estado civil, grau de escolaridade, número de filhos) e sócioocupacional (cargo, tempo de profissão e de trabalho no hospital, turno de trabalho, número de empregos, renda mensal, carga horária semanal) com a finalidade de caracterizar os participantes da pesquisa. 
A pesquisa seguiu as recomendações éticas previstas na Resolução n 466 , de 12 de dezembro de 2012, do Conselho Nacional de Saúde, no que concerne à pesquisa envolvendo seres humanos. A coleta dos dados foi iniciada após se receber autorização da direção do hospital e aprovação do Comitê de Ética em Pesquisa da Universidade Estadual da Paraíba (UEPB), CAAE no 74036817.0.0000.5187. Os instrumentos foram organizados em formato de protocolo e aplicados nos setores hospitalares, conforme a disponibilidade dos participantes, que levaram entre 15 e 20 minutos para concluí-lo.

Os dados foram digitados no programa Statistical Package for the Social Sciences, por meio do qual foram efetuadas estatísticas descritivas (média, frequência, desvio-padrão e porcentagem) para caracterizar a amostra. Foram aplicados o teste Shapiro-Wilk para averiguar a normalidade dos dados e o de correlação ( $\rho$ de Spearman) para verificar a intensidade da relação entre as variáveis. Foi feita a análise de regressão linear múltipla hierárquica para identificar o poder explicativo das variáveis independentes ( $\mathrm{EE}, \mathrm{Cl}$ e IT) sobre a variável dependente ("tensão emocional e depressão"). O nível de significância adotado foi de $p$ menor do que 0,05.

\section{RESULTADOS}

\section{Caracterização da amostra}

Da amostra analisada, 97,3\% eram do sexo feminino, 52,3\% eram casados, 55,5\% tinham filhos e 44,5\% não possuíam filhos. As idades variaram entre 20 e 60 anos $(M=31,95$; $D P=$ $7,90)$, com coeficiente de variação de $25 \%$. O grau de escolaridade predominante foi o ensino médio completo $(45,9 \%)$, seguido de superior incompleto $(26,8 \%)$, pós-graduação $(16,4 \%)$ e superior completo (10,5\%).

Quanto aos dados sócio-ocupacionais, 178 profissionais (80,9\%) eram técnicos de enfermagem e $42(19,1 \%)$ eram enfermeiros. A média de tempo de profissão foi de 8,5 (DP = 7,27 ), com coeficiente de variação de $86 \%$, e a média de tempo de serviço no hospital foi de $5,5$ anos ( $\mathrm{DP}=5,78)$, com coeficiente de variação de $105 \%$. Em relação à jornada de trabalho, $61,4 \%$ exerciam plantão diurno de 6 horas e 38,6\% faziam plantão noturno de 12 horas. Foi observado que $45,5 \%$ tinham também vínculo empregatício em outra instituição de saúde, sendo que $41,8 \%$ exerciam a enfermagem e apenas $2,7 \%$ exerciam outra profissão, porém permanecendo em funções assistenciais, por exemplo, fisioterapia e nutrição. A carga horária semanal variou de 40 horas para quem trabalhava exclusivamente no hospital pesquisado e de 80 horas para quem possuía outro vínculo empregatício. A renda familiar mensal variou entre $\mathrm{R} \$ 1.100,00$ e $\mathrm{R} \$ 10.000,00(\mathrm{M}=3.180,63 ; \mathrm{DP}=1.734,12)$, com coeficiente de variação de $55 \%$.

\section{Saúde mental na amostra}

Para países que ainda não possuem pontos de corte validados nas três subescalas do $\mathrm{MBI}$, Shirom ${ }^{34}$ recomenda aplicar valores iguais ou superiores a 4 ("uma vez por semana") para avaliar as dimensões EE e Cl, e valores iguais ou superiores a 2 ("uma vez ao mês") para avaliar a dimensão IT, uma vez que a subescala dessa dimensão possui itens invertidos. Com base nesse critério, foram calculados os níveis baixos e altos de cada fator do burnout, obtendo-se, respectivamente, 33 sujeitos (15\%) com alto nível de $E E, 2$ sujeitos com elevado $\mathrm{Cl}(0,9 \%)$ e 4 sujeitos com sentimentos de IT $(1,8 \%)$ (Tabela 1$)$.

No QSG-12 (escala de 0 a 3), os resultados são interpretados levando-se em conta que, quanto mais elevada a pontuação no fator "tensão emocional e depressão", mais o indivíduo se sente emocionalmente tenso e esgotado. A mediana encontrada nesse fator foi de 0,40 (percentis $25 \%=0,00 ; 75 \%=0,80$ ), indicando baixos níveis de tensão na amostra, mas, quando se examina a distribuição dos escores por intervalo, foram constatados $88,2 \%$ dos participantes com baixos níveis de tensão emocional, 8,6\% moderadamente tensos e 3,2\% com indícios de depressão (Tabela 2).

Para identificar se houve interação entre as variáveis, foi aplicado o cálculo $\rho$ de Spearman (após o teste Shapiro-Wilk indicar a não normalidade dos dados) entre os escores médios das 
Tabela 1. Burnout em profissionais de enfermagem. Paraíba, Brasil $(N=220)$

\begin{tabular}{lccccc} 
Dimensões de burnout & Níveis baixos & $\%$ & Níveis altos & \% & Freq. \% \\
\hline Exaustão emocional & 187 & 85 & $\mathbf{3 3}$ & $\mathbf{1 5}$ & 100 \\
\hline Cinismo & 218 & 99,1 & $\mathbf{2}$ & $\mathbf{0 , 9}$ & 100 \\
\hline Ineficácia no trabalho & 216 & 98,2 & $\mathbf{4}$ & $\mathbf{1 , 8}$ & 100 \\
\hline
\end{tabular}

Tabela 2. Escores do fator "tensão emocional e depressão" na equipe de enfermagem. Paraíba, Brasil $(\mathrm{N}=220)$

\begin{tabular}{|c|c|c|c|c|c|c|c|c|c|}
\hline \multirow{2}{*}{ Fator QSG-12 } & \multirow{2}{*}{ Mediana } & \multicolumn{2}{|c|}{ Percentis } & \multicolumn{6}{|c|}{ Frequência de participantes por intervalo } \\
\hline & & 25 & 75 & $X<1$ & $\%$ & $1 \leq X<2$ & $\%$ & $\mathbf{X} \geq \mathbf{2}$ & $\%$ \\
\hline $\begin{array}{l}\text { Tensão emocional } \\
\text { e depressão }\end{array}$ & 0,40 & 0,50 & 1,00 & 194 & 88,2 & 19 & 8,6 & 7 & 3,2 \\
\hline
\end{tabular}

variáveis investigadas, sendo os índices de correlação interpretados conforme o critério de classificação de Palant ${ }^{37}$, qual seja: $r=0,10$ a 0,29 representa correlação pequena; $r=0,30$ a 0,49 representa correlação moderada; $r=0,50$ a 1 representa correlação forte. Os resultados mostram que as três dimensões de burnout se correlacionaram significativamente, de forma positiva e moderada, com o fator "tensão emocional e depressão", nos fatores $E E(\rho=0,40 ; p$ $<0,01)$ e Cl $(\rho=0,32 ; \mathrm{p}<0,01)$, e pequena no fator IT $(\rho=0,25 ; \mathrm{p}<0,01)$.

A fim de averiguar o poder preditivo dos fatores de burnout sobre a "tensão emocional e depressão", foi aplicada uma análise de regressão linear múltipla hierárquica (método enter). Os resultados, apresentados nos modelos 1, 2, e 3, evidenciam que houve predição compartilhada entre os três fatores preditores usados na análise (Tabela 3). O fator EE se manteve significativo ( $p<0,001)$ e com maior beta em todas as regressões, sobressaindose como o melhor preditor da "tensão emocional e depressão", explicando sozinho 17\% da variância. Quando foram incluídos na regressão os fatores $\mathrm{Cl}(p<0,001)$ e IT $(p<0,007)$, foi observado que eles se mostraram indicadores significativos, elevando o poder explicativo da "tensão emocional e depressão" para $23 \%$.

\section{DISCUSSÃO}

As características definidoras dos participantes da pesquisa, composta em sua maioria por mulheres, adultas jovens, corroboram outros estudos sobre burnout e/ou depressão em equipes de enfermagem ${ }^{16,27,28,38-43}$. O perfil predominantemente feminino talvez esteja ligado aos primórdios da profissão, com práticas da enfermagem semelhantes ao trabalho doméstico, de cariz caritativo e afetuoso praticado essencialmente por mulheres, apesar do crescente número de homens na profissão atualmente ${ }^{39,40}$.

Semelhante a resultados de outros estudos ${ }^{39,40,44}$, a maioria da amostra possuía ensino médio e era composta por técnicos de enfermagem, sendo tais características condizentes com as configurações adotadas no trabalho da enfermagem e com o Conselho Federal de Enfermagem (COFEN), Resolução n 543/2017, que estabelece os parâmetros para o dimensionamento da equipe de enfermagem, com o maior número de técnicos do que de enfermeiros, conforme o grau de dependência do paciente em relação à equipe de enfermagem ${ }^{45}$.

O trabalho em regimes de turnos constitui uma prática frequente e necessária nos hospitais para que possam funcionar 24 horas ininterruptas. Foi observado que um percentual elevado da amostra $(61,4 \%)$ trabalhava no plantão diurno, corroborando alguns estudos ${ }^{39,40,42,44}$. Esse fato pode ser creditado ao dimensionamento da equipe de enfermagem nos ambientes hospitalares, nos quais durante o dia os pacientes necessitam de mais horas de cuidados da enfermagem do que à noite. 
Tabela 3. Análise de regressão múltipla (linear) para tensão emocional e depressão, tendo como preditores os fatores de burnout. Paraíba, Brasil

\begin{tabular}{|c|c|c|c|c|}
\hline \multirow{3}{*}{ Modelos / preditores } & \multicolumn{4}{|c|}{ Tensão emocional e depressão } \\
\hline & \multirow{2}{*}{ Beta } & \multirow{2}{*}{ Valor-p } & \multicolumn{2}{|c|}{ Intervalo confiança } \\
\hline & & & Limite superior & Limite inferior \\
\hline \multicolumn{5}{|l|}{ Primeiro modelo $\left(r^{2}=0,17\right)$} \\
\hline Constante (desconhecida) & & 0,33 & $-0,07$ & 0,22 \\
\hline Exaustão emocional & 0,41 & 0,0001 & 0,13 & 0,23 \\
\hline \multicolumn{5}{|l|}{ Segundo modelo $\left(r^{2}=0,21\right)$} \\
\hline Constante (desconhecida) & & 0,97 & $-0,16$ & 0,15 \\
\hline Exaustão emocional & 0,32 & 0,0001 & 0,08 & 0,19 \\
\hline Cinismo & 0,21 & 0,002 & 0,04 & 0,18 \\
\hline \multicolumn{5}{|l|}{ Terceiro modelo $\left(r^{2}=23\right)$} \\
\hline Constante (desconhecida) & & 0,22 & $-0,27$ & 0,06 \\
\hline Exaustão emocional & 0,29 & 0,0001 & 0,07 & 0,28 \\
\hline Cinismo & 0,22 & 0,001 & 0,04 & 0,28 \\
\hline Ineficácia no trabalho & 0,16 & 0,007 & 0,02 & 0,15 \\
\hline
\end{tabular}

Sobre os resultados do burnout, foi constatado que a EE foi a única dimensão afetada, atingindo $15 \%$ dos participantes, enquanto as dimensões $\mathrm{Cl}$ e IT foram preservadas, podendose concluir que a SB não incidiu sobre a amostra. A dimensão $E E$, entretanto, foi a primeira que se manifestou na ordem de aparecimento do burnout e antecede a depressão ${ }^{11}$, sendo crucial combatê-la com medidas educativas, psicológicas e de apoio organizacional a fim de conter seu avanço e seu desenrolar para as outras duas fases dentro do processo de adoecimento mental.

De acordo com a literatura ${ }^{9,10,46}$, a EE é a dimensão de burnout que está intimamente ligada à sobrecarga física e psíquica de trabalho, e seu surgimento pode associar-se a valores presentes na construção sócio-histórica das profissões assistenciais ${ }^{47}$. Assim, é possível pensar que em certas profissões, como a enfermagem, ter um papel vocacional e/ou missionário pode conduzir o profissional a assumir cargas extremadas de trabalho, que, consequentemente, exigem gastos elevados de energia psíquica e levam o indivíduo ao completo esgotamento emocional.

Os resultados moderados/altos da "tensão emocional e depressão" em apenas 11,8\% da amostra, epidemiologicamente, não são alarmantes, porém do ponto de vista clínico e organizacional são indesejáveis, pois configuram um processo depressivo em curso, ainda que incipiente, de um dos distúrbios psíquicos mais incapacitantes da atualidade, tanto na esfera de vida pessoal quanto laboral ${ }^{28}$.

Os resultados da correlação sugerem que os profissionais de enfermagem mais esgotados pelo trabalho, com mais dificuldades nas relações interpessoais (clientes e familiares) e com maior sensação de improdutividade são os mais tensos e propensos a desenvolver depressão. A partir das análises de regressão, foram observados $23 \%$ de probabilidade de profissionais com burnout apresentarem sintomas de depressão. E mais, aqueles acometidos somente por elevada $\mathrm{EE}$, que foi a dimensão principal e ponto de partida do desenvolvimento do burnout, além da possibilidade potencial de avançarem na síndrome, apresentaram $17 \%$ de chance de aumento nos níveis de depressão. 
De forma simplificada, é possível concluir que a saúde mental está majoritariamente preservada na amostra, mas a SB está em processo incipiente de desenvolvimento em $15 \%$ dos profissionais de enfermagem, manifestada por altos níveis de EE, que se mostraram preditores mais influentes da depressão.

O critério adotado nesta pesquisa de que burnout predispõe à depressão, e não o inverso, corrobora outros estudos ${ }^{16,26-28,48}$. Com esse critério, foi possível verificar que a $E E$ emergiu como a dimensão nuclear da SB na amostra e que era preditora direta da "tensão emocional e depressão". Esse é um resultado merecedor de atenção psicossocial, visto que profissionais com elevada EE são fortes candidatos a cometer atos de imperícia/iatrogenia (atos ou pensamentos que surtem efeitos negativos ao tratamento do paciente), imprudência e/ou negligência com pacientes e com colegas de trabalho. Tais atos, se recorrentes, desqualificam os serviços hospitalares prestados à população e podem levar o profissional a afastar-se afetivamente dos seus pares, frustrar-se profissionalmente e deprimir-se. Essa situação não é desejável, visto que a enfermagem é uma profissão que prima pela vida, e o hospital onde os participantes trabalham se destaca como um dos principais na variedade de prestação de serviços especializados e em número de atendimentos na cidade de Campina Grande.

Embora não se tenham dados sistematizados sobre as condições de trabalho da amostra, é possível inferir que a EE provenha do fato de que o hospital, por estar situado na segunda maior cidade da Paraíba, atendendo à demanda local, cidades circunvizinhas e outros estados próximos, esteja com alto fluxo de atendimento e internação hospitalar, gerando superlotação e sobrecarregando os profissionais.

A pesquisa apresenta algumas limitações. Uma delas é o fato de ter sido exclusivamente quantitativa e sem coletar dados sobre as condições de trabalho, havendo a necessidade de se investir em estudos que possam acessar as vivências subjetivas dos trabalhadores acerca do cotidiano e conteúdo do trabalho.

Outra limitação é que foi desenvolvida dentro de um contexto hospitalar muito específico, composto exclusivamente por profissionais da enfermagem, quando se sabe que eles trabalham em equipe de assistência multiprofissional. Essa limitação, entretanto, abre espaço para que outros estudos sobre a temática sejam realizados com amostra mais ampla de profissionais de saúde. Também o fato de ter sido realizado com uma amostra não probabilística exige cautela para que seus achados não sejam generalizados para outros contextos ou realidades hospitalares.

Mesmo com as limitações, os resultados da pesquisa indicam a necessidade de intervenções que mobilizem gestores e trabalhadores para a busca de alternativas práticas para conter a EE e os possíveis quadros depressivos dentro do hospital, preservando a qualidade da saúde e do trabalho dos enfermeiros. Tais intervenções podem incluir medidas para: identificar as necessidades e expectativas dos profissionais; criar espaços de discussão e reflexão de conflitos, buscando soluções; implementar programas de apoio psicossociológico aos profissionais; propiciar cursos de capacitação permanente para atender aos Procedimentos Operacionais Padrão (POP) específicos de cada setor hospitalar, evitando possíveis acidentes e iatrogenias; proporcionar condições de repouso e de alimentação adequadas a cada plantão; incentivar práticas de exercícios físicos; flexibilizar horários e fornecer incentivos salariais aos trabalhadores que investem na própria capacitação profissional.

\section{REFERÊNCIAS}

1. Costa MTP, Borges LDO, Barros SC. Condições de trabalho e saúde psíquica: um estudo em dois hospitais universitários. Rev Psi Org Trab. 2015;15(1):43-58. http://dx.doi.org/10.17652/rpot/2015.1.490.

2. Amazarray MR, Câmara SG, Carlotto MS Investigação em saúde mental e trabalho no âmbito da saúde pública no Brasil. In: Crespo AR, Bottega CG, Perez KV, editores. Atenção à saúde mental do trabalhador: sofrimento e transtornos psíquicos relacionados ao trabalho. Porto Alegre: Evangraf; 2014.

3. Carvalho DP, Rocha LP, Barlem JGT, Dias JS, Schallenberger CD. Cargas de trabalho e a saúde do trabalhador de enfermagem: revisão integrativa. Cog Enf. 2017;22(1):1-11. http://dx.doi.org/10.5380/ce.v22i1.46569. 
4. Fonseca MLG, Sá MDC. A insustentável leveza do trabalho em saúde: excessos e invisibilidade no trabalho da enfermagem em oncologia. Saúde Debate. 2015;39(spe):298-306. http://dx.doi.org/10.5935/01031104.20155005247.

5. Machado $M H$, Santos MR, Oliveira E, Wermelinger M, Vieira M, Lemos W, et al. Condições de trabalho da enfermagem. Enferm Foco. 2016;7(esp):63-71. http://dx.doi.org/10.21675/2357-707X.2016.v7.nESP.695.

6. Alvim CCE, Souza MMT, Gama LN, Passos JP. Relação entre processo de trabalho e adoecimento mental da equipe de enfermagem. Rev Flu Exten Univ [Internet]. 2017;7(1):12-6 [citado em 2018 Set 3]. Disponível em: http://editorauss.uss.br/index.php/RFEU/article/view/918

7. Andrade AL, Moraes TD, Tosoli AM, Wachelke J. Burnout, clima de segurança e condições de trabalho em profissionais hospitalares. Rev Psi Org Trab. 2015;15(3):233-45. http://dx.doi.org/10.17652/rpot/2015.3.565.

8. Silva RP, Barbosa SC, Silva SS, Patrício DF. Burnout e estratégias de enfrentamento em profissionais de enfermagem. Arq Bras Psicol. 2015;67(1):130-45.

9. Maslach C. Job burnout: new directions in research and intervention. Curr Dir Psychol Sci. 2003;5(13):18992. http://dx.doi.org/10.1111/1467-8721.01258.

10. Maslach C. Comprendiendo el burnout. Rev Cien Trab. 2009;11(32):37-43.

11. Maslach C, Schaufeli WB, Leiter MP. Job burnout. Annu Rev Psychol. 2001;52(1):397-422. http://dx.doi. org/10.1146/annurev.psych.52.1.397. PMid:11148311.

12. Gil-Monte PR. El síndrome de quemarse por el trabajo (burnout) como fenómeno transcultural. Inf Psicol. 2008;91(1):4-11.

13. Santos CS. Fatores da síndrome de burnout como preditores da depressão em policiais militares [trabalho de conclusão de curso]. Campina Grande: Universidade Estadual da Paraíba; 2016.

14. Brasil. Ministério da Saúde. Organização Pan-Americana da Saúde no Brasil. Doenças relacionadas ao trabalho: manual de procedimentos para os serviços de saúde. Brasília: Ministério da Saúde do Brasil; 2001.

15. Manetti ML. Estudos de aspectos profissionais e psicossociais no trabalho e a depressão em enfermeiros atuantes em ambiente hospitalar [tese]. Ribeirão Preto: Universidade de São Paulo; 2009 [citado em 2018 Set 3]. Disponível em: http://www.teses.usp.br/teses/disponiveis/22/22132/tde-10032010-083634/en.php

16. Murcho N, Jesus SN, Pacheco E. A relação entre a depressão em contexto laboral e o burnout: um estudo empírico com enfermeiros. Psicol Saude Doencas [Internet]. 2009;10(1):57-68 [citado em 2018 Set 3]. Disponível em: http://www.scielo.mec.pt/scielo.php?script=sci_arttext\&pid=S1645-00862010000100003

17. Vargas D, Dias APV. Prevalência de depressão em trabalhadores de enfermagem de unidade de terapia intensiva: estudo em hospitais de uma cidade do noroeste do Estado São Paulo. Rev Latino-Am Enfermagem [Internet]. 2011;19(5):1-9 [citado em 2018 Set 3]. Disponível em: http://www.scielo.br/pdf/ rlae/v19n5/pt_08.pdf

18. World Health Organization. Depression, let's talk campaign essentials World Health Day [Internet]. Geneva:WHO; 2017 [citado em 2018 Set 3]. Disponível em: http://www.who.int/campaigns/world-healthday/2017/campaign-essentials/en/

19. Mallar SC, Capitão CG. Burnout e hardiness: um estudo de evidência de validade. Psico-USF. 2004;9(1):1929. http://dx.doi.org/10.1590/S1413-82712004000100004.

20. Pacheco ACCL. Ansiedade, depressão, stresse, estratégias de coping, suporte social em enfermeiros que trabalham por turnos e em regime de horário fixo diurno [dissertação]. Lisboa: Universidade Lusófona de Humanidades e Tecnologias; 2013. [citado em 2018 Set 3]. Disponível em: http://recil.grupolusofona. pt/handle/10437/4996

21. Varoli I, Souza CB. O sofrimento dos que tratam: burnout em profissionais de saúde mental. In: Pais Ribeiro JL, Isabel L, editors. Actas $5^{\circ}$ Congresso Nacional de Psicologia da Saúde; 2004; Lisboa. Lisboa: Instituto Superior de Psicologia Aplicada; 2004. p. 691-7.

22. Arice G, Batista MN, Morais PR, Souza FG, Reis MS. Correlação entre sintomatologia depressiva e burnout em um grupo de servidores públicos. Psicol. Argum. 2004;22(37):53-62.

23. Batista MN, Morais PR, Carmo NC, Souza GO, Cunha AF. Avaliação da depressão, síndrome de burnout, e qualidade de vida em bombeiros. Psicol. Argum. 2005;23(42):47-54. http://dx.doi.org/10.7213/rpa. v23i42.20009.

24. Gil-Monte P, Peiró JM. Desgaste psíquico en el trabajo: el síndrome de quemarse. Madrid: Editorial Síntesis; 1997. 
25. Trejo-Lucero H, Torres-Pérez J, Valdivia-Chávez M. Asociación entre síndrome de burnout y depresión en personal de enfermería que labora en un hospital de alta especialidad del Estado de México. Arch Inv Mat Inf [Internet]. 2011;3(1):44-7 [citado em 2018 Set 3]. Disponível em: http://www.medigraphic.com/ pdfs/imi/imi-2011/imi111i.pdf

26. Vasconcelos EM, Martino MMF, França SPS. Burnout e sintomatologia depressiva em enfermeiros de terapia intensiva: análise de relação. Rev Bras Enferm. 2018;71(1):147-53. PMid:29324955.

27. Quintas S, Queirós C, Marques A, Orvalho V. Os enfermeiros e a sua saúde no trabalho: a relação entre depressão e burnout. Int J Work Conditions [Internet]. 2017;(13):1-20 [citado em 2018 Set 3]. Disponível em: https://repositorio-aberto.up.pt/handle/10216/106532

28. Schonfeld I, Bianchi R. Burnout and depression: two entities or one? J Clin Psychol. 2016;72(1):22-37. http://dx.doi.org/10.1002/jclp.22229. PMid:26451877.

29. Sarriá A, Guardiã J, Freixa M. Introducción a la estadística en Psicología. Barcelona: Ediciones de la Universitat de Barcelona; 1999.

30. Maslach C, Jackson SE. Maslach burnout Inventory: the measurement of experienced burnout. J Occup Behav. 1981;2(1):99-113. http://dx.doi.org/10.1002/job.4030020205.

31. Benevides-Pereira AMT. MBI - Maslach Burnout Inventory e suas adaptações para o Brasil. In: Anais da $32^{a}$ Reunião Anual de Psicologia; 2001; Rio de Janeiro. Ribeirão Preto: SBP; 2001. p. 84-5.

32. Carlotto MS, Câmara SG. Propriedades psicométricas do Maslach Burnout Inventory em amostra multifuncional. Estud Psicol [Internet]. 2007;24(1):325-32 [citado em 2018 Set 3]. Disponível em: http:// www.scielo.br/scielo.php?script=sci_arttext\&pid=S0103-166X2007000300004

33. Tamayo MR. Relação entre a síndrome de burnout e os valores organizacionais no pessoal de enfermagem de dois hospitais públicos [dissertação]. Brasília: Universidade de Brasília; 1997.

34. Shirom A. Burnout in work organizations. In: Cooper CL, Robertson I, editores. International review of industrial and organizational psychology. New York: Wiley; 1989. p. 25-48.

35. Goldberg DP. The detection of psychiatric illness by questionnaire.Londres: Oxford University Press; 1972.

36. Borges LO, Argolo JCT. Estratégias organizacionais na promoção da saúde mental do indivíduo podem ser eficazes? In: Jacques G, Codo W, editores. Saúde mental e trabalho: leituras. Petrópolis: Vozes; 2002. p. 271-5.

37. Palant J. SPSS: survical manual. London: McGraw-Hill; 2007. p. 132-3.

38. Ferreira NN, Lucca SR. Síndrome de burnout em técnicos de enfermagem de um hospital público do estado de São Paulo. Rev Bras Epidemiol. 2015;18(1):68-79. http://dx.doi.org/10.1590/1980-5497201500010006.

39. Meneghini F, Paz AA, Lautert L. Fatores ocupacionais associados aos componentes da síndrome de burnout em trabalhadores de enfermagem. Texto Contexto Enferm. 2011;20(2):225-33. http://dx.doi.org/10.1590/ S0104-07072011000200002.

40. Pereira DR. Sindrome de burnout em profissionais de enfermagem: um estudo psicossociológico [dissertação]. João Pessoa: Universidade Federal da Paraíba; 2014.

41. Oliveira V, Pereira T. Ansiedade, depressão e burnout em enfermeiros: Impacto do trabalho por turnos. Rev Enf Ref. 2012;3(7):43-54. http://dx.doi.org/10.12707/RIII1175.

42. Trigo TR. Validade fatorial do Maslach Burnout Inventory - Human Services Survey (MBI-HSS) em uma amostra Brasileira de auxiliares de enfermagem de um hospital universitário: influencia da depressão [dissertação]. São Paulo: USP; 2010 [citado em 2018 Set 3]. Disponível em: http://www.teses.usp.br/teses/ disponiveis/5/5142/tde-26052011-123120/pt-br.php

43. Ferreira GB, Aragão AEA, Oliveira PS. Síndrome de burnout na enfermagem hospitalar/intensivista: o que dizem os estudos? Sanare [Internet]. 2017;16(1):100-8 [citado em 2018 Set 3]. Disponível em: https:// sanare.emnuvens.com.br/sanare/article/view/1100

44. Andrade T, Hoch REE, Vieira KM, Rodrigues CMC. Síndrome de burnout e suporte social no trabalho: a percepção dos profissionais de enfermagem de hospitais públicos e privados. Rev Organ Soc. 2012;19(61):231-51. http://dx.doi.org/10.1590/S1984-92302012000200004.

45. Brasil. Conselho Federal de Enfermagem - COFEN. Resolução Cofen n 543 de 18 de Abril de 2017 Atualiza e estabelece parâmetros para o dimensionamento do quadro de profissionais de enfermagem nos serviços/locais em que são realizadas atividades de enfermagem. Diário Oficial da União [Internet], Brasília, 8 de maio de 2017 [citado em 2018 Set 3]. Disponível em: http://www.cofen.gov.br/resolucaocofen-5432017_51440.html

46. Maslach C. A multidimensional theory of burnout. In: Cooper GL, editor. Theories of organizational stress. Oxford: Oxford University Press; 1998. p. 68-85. 
47. Batista JBV, Carlotto MS, Coutinho AS, Augusto LGS. Prevalência da síndrome de burnout e fatores sociodemográficos e laborais em professores de escolas municipais da cidade de João Pessoa, PB. Rev Bras Epidemiol. 2010;13(3):502-12. http://dx.doi.org/10.1590/S1415-790X2010000300013. PMid:20857036.

48. Cavalcanti IL, Lima FLT, Souza TA, Silva MJS. Burnout e depressão em residentes de um programa multiprofissional em oncologia: estudo longitudinal prospectivo. Rev Bras Educ Med. 2018;42(1):188-96. http://dx.doi.org/10.1590/1981-52712018v42n1rb20170078. 